\title{
Origin-independent plasmid replication occurs in vaccinia virus cytoplasmic factories and requires all five known poxvirus replication factors
}

\author{
Frank S De Silva and Bernard Moss*
}

Address: Laboratory of Viral Diseases, National Institute of Allergy and Infectious Diseases, National Institutes of Health, Bethesda, Maryland 20892-0445, USA

Email: Frank S De Silva - fdesilva@niaid.nih.gov; Bernard Moss* - bmoss@nih.gov

* Corresponding author

Published: 22 March 2005

Virology Journal 2005, 2:23 doi:10.1 186/1743-422X-2-23

This article is available from: http://www.virologyj.com/content/2/1/23

(c) 2005 De Silva and Moss; licensee BioMed Central Ltd.

This is an Open Access article distributed under the terms of the Creative Commons Attribution License (http://creativecommons.org/licenses/by/2.0), which permits unrestricted use, distribution, and reproduction in any medium, provided the original work is properly cited.
Received: 10 March 2005

Accepted: 22 March 2005

\begin{abstract}
Background: Replication of the vaccinia virus genome occurs in cytoplasmic factory areas and is dependent on the virus-encoded DNA polymerase and at least four additional viral proteins. DNA synthesis appears to start near the ends of the genome, but specific origin sequences have not been defined. Surprisingly, transfected circular DNA lacking specific viral sequences is also replicated in poxvirus-infected cells. Origin-independent plasmid replication depends on the viral DNA polymerase, but neither the number of additional viral proteins nor the site of replication has been determined.
\end{abstract}

Results: Using a novel real-time polymerase chain reaction assay, we detected a $>400$-fold increase in newly replicated plasmid in cells infected with vaccinia virus. Studies with conditional lethal mutants of vaccinia virus indicated that each of the five proteins known to be required for viral genome replication was also required for plasmid replication. The intracellular site of replication was determined using a plasmid containing 256 repeats of the Escherichia coli lac operator and staining with an E. coli lac repressor-maltose binding fusion protein followed by an antibody to the maltose binding protein. The lac operator plasmid was localized in cytoplasmic viral factories delineated by DNA staining and binding of antibody to the viral uracil DNA glycosylase, an essential replication protein. In addition, replication of the lac operator plasmid was visualized continuously in living cells infected with a recombinant vaccinia virus that expresses the lac repressor fused to enhanced green fluorescent protein. Discrete cytoplasmic fluorescence was detected in cytoplasmic juxtanuclear sites at $6 \mathrm{~h}$ after infection and the area and intensity of fluorescence increased over the next several hours.

Conclusion: Replication of a circular plasmid lacking specific poxvirus DNA sequences mimics viral genome replication by occurring in cytoplasmic viral factories and requiring all five known viral replication proteins. Therefore, small plasmids may be used as surrogates for the large poxvirus genome to study trans-acting factors and mechanism of viral DNA replication. 


\section{Background}

Vaccinia virus (VAC), the prototype for the family Poxviridae, is a large double-stranded DNA virus that encodes numerous enzymes and factors needed for RNA and DNA synthesis, enabling it to replicate in the cytoplasm of infected cells [1]. More than 20 viral proteins including a multi-subunit RNA polymerase and stage specific transcription factors are involved in viral RNA synthesis [2]. Genetic and biochemical studies identified five viral proteins essential for viral DNA replication, namely the viral DNA polymerase [3-8], polymerase processivity factor $[9,10]$, DNA-independent nucleoside triphosphatase [1113], serine/threonine protein kinase [14-17], and uracil DNA glycosylase [18-21]. In addition, the virus encoded Holliday junction endonuclease is required for the resolution of DNA concatemers into unit-length genomes [22]. Other proteins that may contribute to viral DNA replication, include DNA type I topoisomerase, single stranded DNA binding protein, DNA ligase, thymidine kinase, thymidylate kinase, ribonucleotide reductase and dUTPase (reviewed in reference [1]).

The VAC genome consists of a $192 \mathrm{kbp}$ linear duplex DNA with covalently closed hairpin termini $[23,24]$. A model for poxvirus DNA replication begins with the introduction of a nick near one or both ends of the hairpin termini, followed by polymerization of nucleotides at the free 3 '-OH end, strand displacement and concatemer resolution $[25,26]$. Nicking is supported by changes in the sedimentation of the parental DNA following infection, and labeling studies suggested that replication begins near the ends of the genome $[27,28]$. Efforts to locate a specific origin of replication in the VAC genome led to the surprising conclusion that any circular DNA replicated as head-totail tandem arrays in cells infected with VAC [29,30]. Origin-independent plasmid replication was also shown to occur in the cytoplasm of cells infected with other poxviruses including Shope fibroma virus and myxoma virus as well as with African swine fever virus [30,31]. In contrast, studies with linear minichromosomes containing hairpin termini provided evidence for cis-acting elements in VAC DNA replication [32]. It was considered that plasmid replication might be initiating non-specifically, perhaps at random nicks in DNA.

Although transfected plasmids were used to study the resolution of poxvirus concatemer junctions [33-37], the system has not been exploited for studies of viral DNA synthesis. The goal of the present study was to determine how closely plasmid replication mimics viral genome replication. For example, if some viral proteins are needed for initiating DNA synthesis at specific origins near the ends of the viral genome, they might not be required for plasmid replication. In addition, we were curious as to whether synthesis of plasmid DNA occurs diffusely in the cytoplasm, since the transfected DNA enters cells independently of virus and contains no viral targeting sequences. Contrary to these speculations, we found that each of the five viral proteins known to be required for viral genome replication was needed for origin-independent replication of plasmids. Moreover, both plasmid and genome replication occurred in discrete viral cytoplasmic factory areas. Thus, small circular plasmids are useful surrogates for the large viral genome in studying the mechanism of poxvirus DNA replication and the trans-acting factors required.

\section{Results}

Determination of plasmid replication by real-time PCR

The replication of plasmids and linear minichromosomes, which were transfected into cells infected with VAC, was previously demonstrated by autoradiography following hybridization of ${ }^{32} \mathrm{P}$-labeled probes to Southern blots [29,30,32]. Methylated input DNA prepared in E. coli was distinguished from unmethylated DNA replicated in infected mammalian cells by digestion with $D p n \mathrm{I}$ and MboI, which cleave GmATC and GATC sequences, respectively. DeLange and McFadden [30] had reported an 8fold net increase of a circular plasmid lacking viral sequences in rabbit cells infected with myxoma virus, whereas Du and Traktman [32] had seen a 2-fold net increase of a linear minichromosome containing VAC genome termini in mouse $\mathrm{L}$ cells infected with VAC, but a much lower increase of a circular plasmid lacking viral sequences. We compared the replication of three types of DNA (super coiled circular, linear, and linear minichromosome) in African green monkey BS-C-1 cells, which has become a standard cell line for VAC research. Southern blot analysis of the DpnI-digestion products of DNA isolated from cells infected with VAC and transfected with super coiled pUC13 revealed a prominent high molecular weight band migrating above the 23.1 -kbp marker, presumably representing head-to-tail concatemers (Fig. 1A). A prominent DpnI-resistant band, migrating between the 4.4 and $6.6 \mathrm{kbp}$ markers, was obtained by digestion of DNA from infected BS-C-1 cells transfected with the covalently closed minichromosome. However, only small digestion products were obtained upon DpnI-treatment of DNA from cells transfected with linear pUC13. In addition, DpnI-resistant bands were not detected by digestion of DNA from mock-infected cells transfected with a linear minichromosome or 10 times more super coiled plasmid (Fig. 1A). This experiment confirmed the need for VAC infection and either a circular plasmid or a linear minichromosome template for DNA replication. Moreover, we did not see greater replication of the linear minichromosome than the circular plasmid as had been reported (32).

To improve quantification of plasmid replication and to establish a non-radioactive method for rapid analysis of 
A

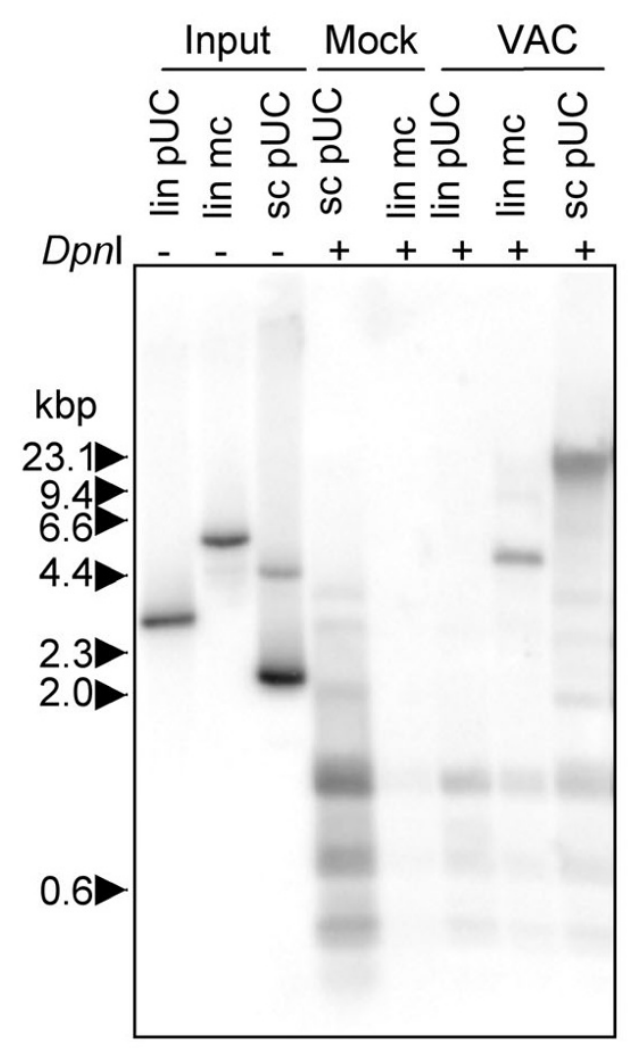

B
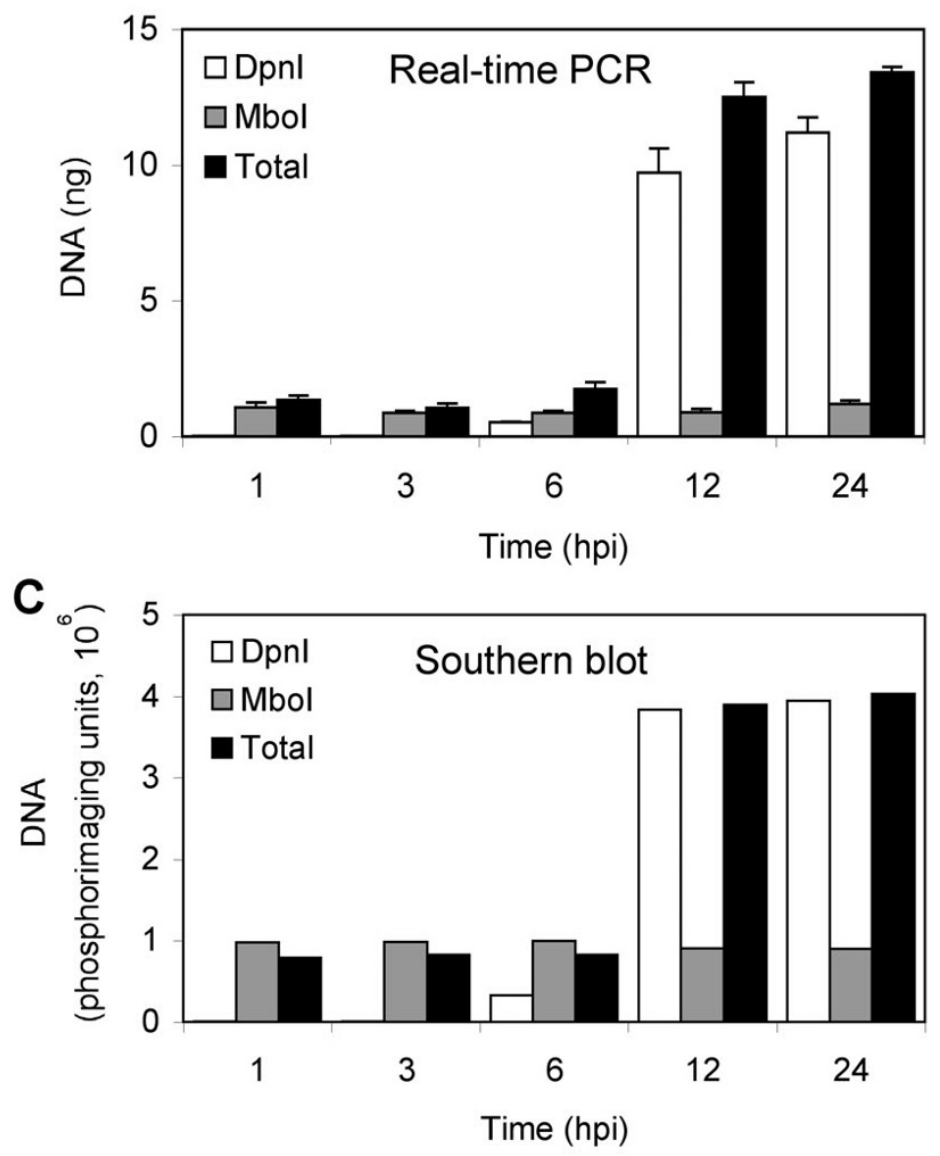

Figure I

Replication of transfected DNA in VAC-infected cells. (A) Southern blot of replicated circular plasmid and linear minichromosome. B-SC-I cells were infected with VAC and I h later transfected with equal molar amounts (20 fmol) of super coiled pUCI 3 (sc pUC), pUCI 3 linearized by digestion with EcoRI (lin pUC), linear minichromosome containing pUCI3 and I.3 kbp viral telomeric sequences (lin $\mathrm{mc}$ ). As a control, cells were mock infected and transfected with 20 fmol of linear minichromosome or 10 times that amount $(200 \mathrm{fmol})$ of super coiled pUCI3. At $24 \mathrm{~h}$ after infection or mock infection, cells were collected and total DNA extracted. Total DNA $(2 \mu \mathrm{g})$ was digested with Dpnl subjected to agarose gel electrophoresis and analyzed by Southern blot hybridization using a $32 \mathrm{P}$-labeled pUCI3 probe. Samples $(0.5 \mathrm{fmol}$ of lin pUC, $0.5 \mathrm{fmol}$ of lin mc, I fmol sc pUC) of the DNA used for transfections (input DNA) were also analyzed. The positions of marker DNA (kbp) are shown on the left. (B) Real-time PCR of replicated plasmid. BS-C-I cells were transfected with the plasmid p7I6 at $24 \mathrm{~h}$ prior to infection with VAC. At indicated hours post infection (hpi), cells were harvested and total DNA extracted. DNA was untreated or treated with Dpnl or Mbol and analyzed by real-time PCR using primers specific to plasmid DNA. (C) Quantification of Southern blot. DNA described in panel $(B)$ was digested with EcoRI prior to Mbol or Dpnl treatment. The digested DNA samples were subjected to gel electrophoresis, transferred to a Nylon membrane, hybridized to a $32 \mathrm{P}$-labeled p7I6 probe, and the radioactivity quantified with a phospholmager.

multiple samples, we devised a real-time PCR assay using primers 152 bp apart that flanked two DpnI/MboI sites in a circular plasmid lacking VAC DNA sequences. In initial experiments, we followed the protocol of previous studies by transfecting the plasmid after infection [29,30]. However, MboI-resistant input DNA as well as DpnI-resistant replicated DNA increased with time, suggesting that entry of DNA into the cell occurred continuously even though the medium was changed at $4 \mathrm{~h}$ (data not shown). To avoid this problem in subsequent experiments, DNA was transfected $24 \mathrm{~h}$ prior to infection. Total DNA was isolated at various times, digested with $D p n I, M b o I$, or left uncut 
and subjected to real-time PCR. Under these conditions, MboI-resistant DNA did not increase, whereas DpnI-resistant DNA increased $\sim 18$ fold between 3 and $6 \mathrm{~h}$ and $\sim 400$ fold by $24 \mathrm{~h}$ (Fig. 1B). Moreover, total DNA increased $\sim 10$ fold. Increased DpnI-resistant DNA was not detected in mock-infected cells (data not shown).

Previous Southern blotting studies had indicated that plasmid replication paralleled genome replication [30]. We compared the kinetics of plasmid replication obtained by real-time PCR with Southern blotting. For the latter analysis, total DNA was first digested with EcoRI to resolve head-to-tail concatemers into linear units followed by digestion with MboI or DpnI. After electrophoresis, the DNA was transferred to a nylon membrane, hybridized to a 32P-labeled plasmid probe, and the amount of DNA quantified using a PhosphorImager. The DpnI-resistant and total DNA increased with time, whereas the MboIresistant DNA did not (Fig. 1C). The Southern blot analysis suggested that the amount of replicated plasmid plateaued after $12 \mathrm{~h}$, whereas it continued to increase slightly as determined by PCR (Fig. 1B), suggesting that the latter method has the greater dynamic range as well as being more convenient.

\section{Determination of the trans-acting factors required for plasmid replication}

The dependence of VAC genome replication on expression of five viral early proteins was previously determined by analysis of conditional lethal mutants. Because of the absence of cis-acting VAC DNA sequences, we considered that plasmid replication might only mimic DNA elongation steps and therefore require only a subset of viral proteins. To test this hypothesis, the plasmid was transfected into BS-C-40 cells (a derivative of BS-C- 1 cells that have been passaged at $40^{\circ} \mathrm{C}$ ), which were subsequently infected with a VAC ts mutant under permissive and nonpermissive conditions. Plasmid replication was quantified by real-time PCR. Wild type VAC strain WR and Cts16, which has a mutation in the I7 gene encoding a protease required for VAC morphogenesis but not DNA synthesis [38], served as positive controls. Plasmid DNA synthesis was higher at $39.5^{\circ} \mathrm{C}$ than $31^{\circ} \mathrm{C}$ for both WR and Cts 16 (Fig. 2A). In contrast, the reverse was true for each mutation known to impair DNA replication at the non-permissive temperature. Indeed, plasmid replication was barely detected at $39.5^{\circ} \mathrm{C}$ in cells infected with Cts24, Cts42, and ts185, which have defects in the D5 nucleoside triphosphatase, the E9 DNA polymerase, and the A20 processivity factor (Fig. 2A). The reduction in plasmid replication was less complete at $39.5^{\circ} \mathrm{C}$ in cells infected with Cts25, which has a defect in the $\mathrm{B} 1$ serine/threonine protein kinase, which is consistent with previous observations that showed viral genome accumulation was only moderately reduced in BS-C-40 cells at non-permissive tempera- tures [15]. The relatively low replication of plasmid at $31^{\circ} \mathrm{C}$ in cells infected with Cts42 and ts 185 (Fig. 2A) suggested that the mutated DNA polymerase and processivity factor were still somewhat defective even under "permissive" conditions.

Previous studies had shown that expression of VAC uracil DNA glycosylase was required for genome replication [39]. To determine whether this protein is required for plasmid replication, we used mutant virus vD4-ZG, in which the uracil DNA glycosylase gene was deleted [39], and rabbit cell lines lacking (RK-13) or stably expressing (RKD4R) VAC uracil DNA glycosylase [39]. We found that plasmid DNA replication was only detected in the cell line stably expressing the viral uracil DNA glycosylase (Fig. $2 \mathrm{~B}$ ), indicating a requirement for this protein as well as each of the other four factors.

\section{Transfected plasmid DNA accumulates in viral factories}

VAC genomic DNA accumulates in specialized cytoplasmic factory areas near the nucleus. However, the intracytoplasmic location of plasmid replication had not been determined. We needed a specific tag to distinguish viral and plasmid DNA in order to locate the latter in infected cells. Several studies have used multimerized E. coli lac operator (lacO) binding sites and lac repressor (lacI) fusion protein interactions to examine chromatin organization and chromosome dynamics in living cells [40-43]. To apply this strategy, we transfected cells with a $10.5 \mathrm{kbp}$ plasmid pSV2-dhfr-8.32 [44] containing 256 lacO repeats and infected the cells $24 \mathrm{~h}$ later. Initial experiments confirmed that plasmid replication occurred following VAC infection as described above for smaller plasmids (data not shown). Next we transfected cells with pSV2-dhfr8.32 and then infected them with vV5D4, a recombinant VAC that expresses V5 epitope-tagged uracil DNA glycosylase. At $12 \mathrm{~h}$ after infection, DNA in the nucleus and cytoplasmic factories was visualized by Hoechst staining (Fig. 3 ). The plasmid appeared to be excluded from the nucleus and present exclusively in cytoplasmic viral factories as determined by staining the cells with a maltose binding protein (MBP)-lacI fusion protein and an antibody to MBP (Fig. 3). In addition, the plasmid sites contained the VAC DNA glycosylase, as shown by staining with antibody to the V5 tag of the latter protein (Fig. 3). No MBP staining was detected when a control plasmid lacking lac $\mathrm{O}$ sequences was transfected (data not shown).

\section{Visualization of replicating plasmid DNA in live cells}

In the above experiment, the cells were fixed and stained in order to visualize the plasmid DNA. We considered that these steps might be avoided by expressing a GFP-lacI fusion protein with a nuclear localization signal (NLS). The GFP tag allowed visualization of lacI by fluorescence microscopy while the NLS served to translocate GFP-lacI, 
A

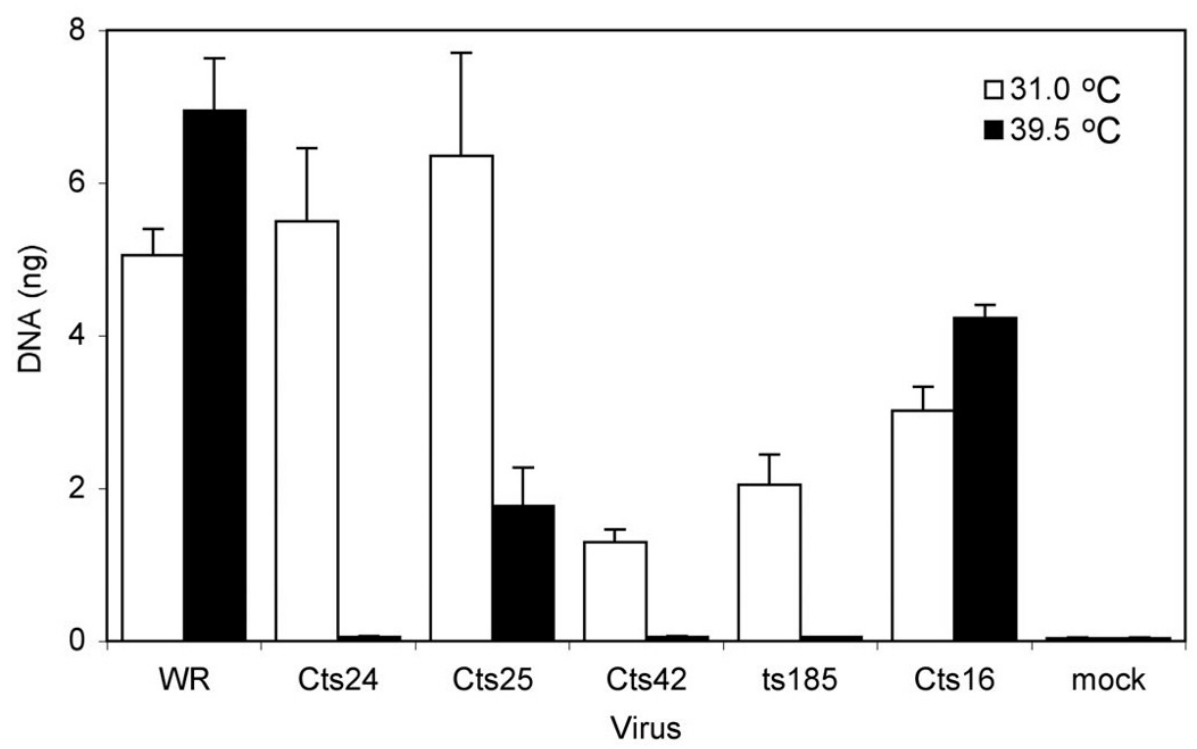

B

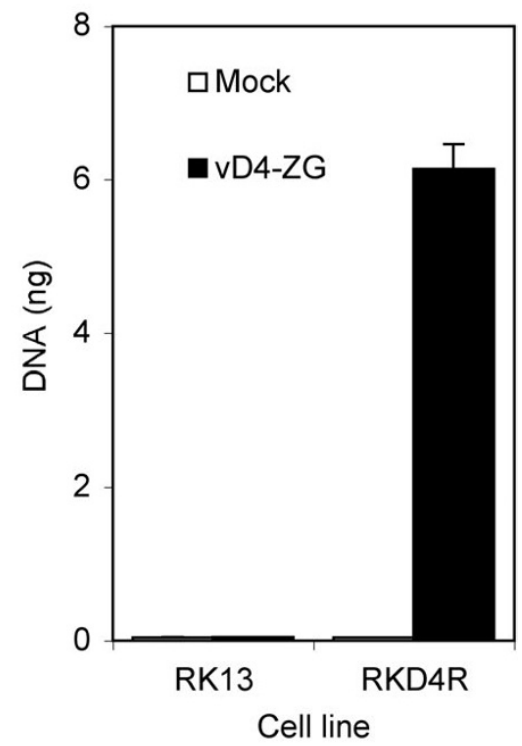

Figure 2

Viral protein requirements for plasmid replication. (A) Conditional lethal ts mutants. BS-C-40 cells were transfected with p7I 6 and $24 \mathrm{~h}$ later were mock infected or infected with 3 PFU per cell of wild type VAC (WR) or ts mutant Cts 24 , Cts 25 , ts 185 , or $\mathrm{Cts} 16$ at permissive $\left(3 \mathrm{I}^{\circ} \mathrm{C}\right)$ and non-permissive $\left(39.5^{\circ} \mathrm{C}\right)$ temperatures for $24 \mathrm{~h}$. DNA was then isolated, digested with $D p n \mathrm{l}$ and quantified by real-time PCR. (B) D4R deletion mutant. RKD4R and RK-I 3 cells were transfected with p7I6 and $24 \mathrm{~h}$ later were infected with 3 PFU of vD4-ZG. At 24 after infection, DNA was isolated, digested with Dpnl, and the amount of DNA quantified by real-time PCR.

which was not specifically bound to lacO sequences in DNA, from the cytoplasm to the nucleus. In order to express the fusion protein prior to and during DNA replication, we constructed the recombinant vGFP-lacI with the open reading frame encoding the GFP-lacI-NLS fusion protein regulated by a viral early/late promoter. HeLa cells were transfected with pSV2-dhfr-8.32 and infected $24 \mathrm{~h}$ later with vGFP-lacI. Bright green fluorescence was detected over the viral factory areas and nuclei, which correlated with Hoechst staining (Fig. 4). In cells with multiple viral factories, however, not every one exhibited green fluorescence. The viral factory regions were also visualized by staining with an antibody to viral RNA polymerase, which surrounded and included the DNA sites at $12 \mathrm{~h}$ after infection (Fig. 4). When a control plasmid (p716) lacking lac $\mathrm{O}$ sites was transfected, the green fluorescence was strictly localized to the nucleus (Fig. 4).

Having established the specificity of the GFP-lacI binding by co-localization, we examined fluorescence of live cells by time-lapse microscopy following transfection with pSV2-dhfr-8.32 and infection with vGFP-lacI. Weak GFP fluorescence was detected at about $5.5 \mathrm{~h}$ after infection (not shown) and was largely over the nucleus, reflecting the targeting due to the NLS. A region of juxtanuclear fluorescence corresponding to a viral factory was seen clearly at $7.5 \mathrm{~h}$ and over the next several hours increased in intensity (Fig. 5). The time course suggested that the factory region was the site of replication as well as accumulation of the plasmid DNA.

\section{Discussion}

The replication of circular DNA lacking viral sequences as head-to-tail concatemers in the cytoplasm of cells infected with a poxvirus was reported nearly 20 years ago [29,30]. Fortuitous poxviral origins were ruled out by the replication of 5 different circular DNAs and no evidence was obtained for integration into the viral genome by nonhomologous recombination. These data strongly suggested autonomous plasmid replication by a rolling circle or theta mechanism. The significance of sequence nonspecific DNA replication was called into question by Du and Traktman [32], who reported only low-level replication of a super coiled plasmid compared to a linear minichromosome containing specific telomere sequences [32]. However, our determination of a 10-fold increase in net plasmid DNA compares favorably to the 2-fold increase achieved with the most efficient 

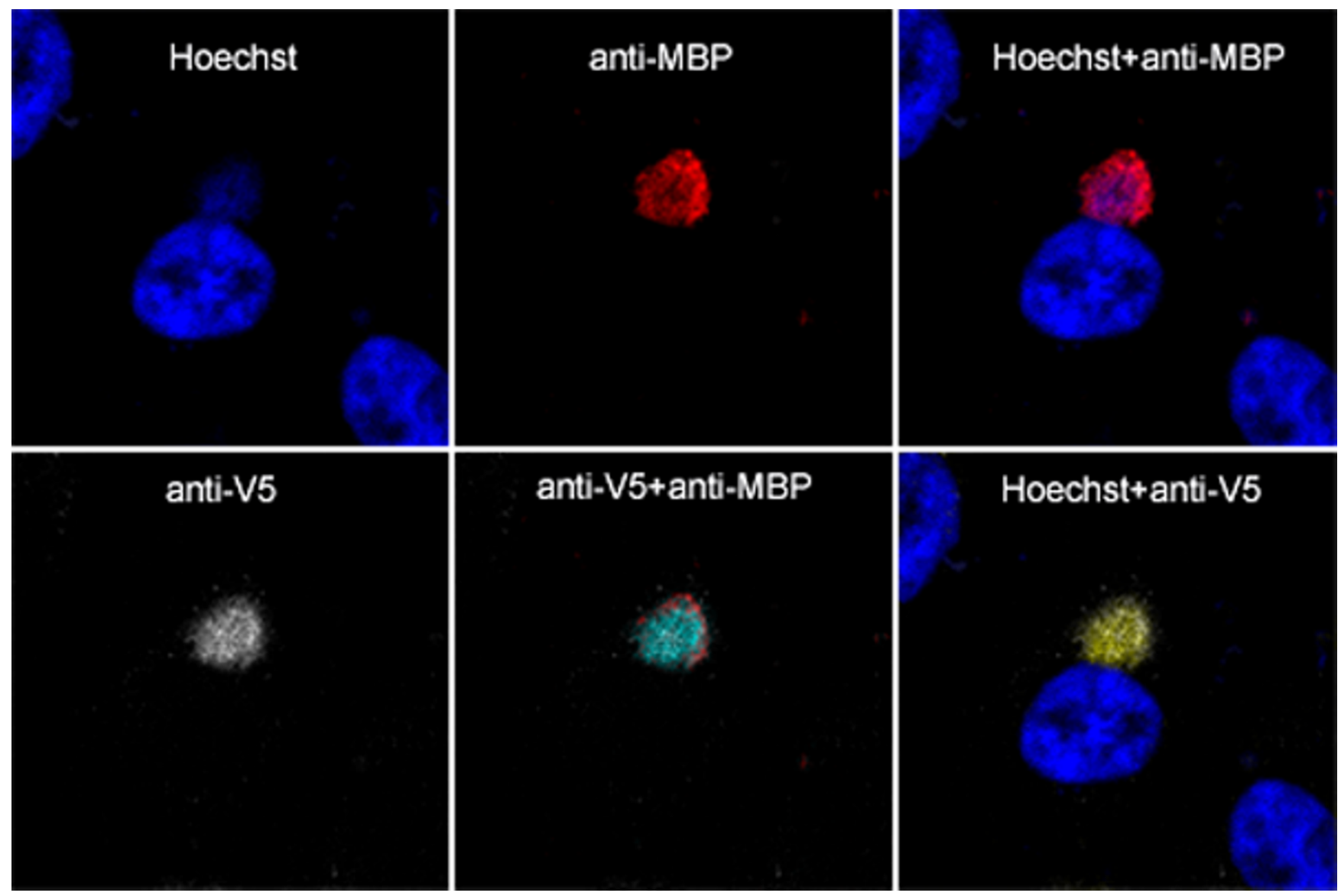

Figure 3

Intracellular localization of replicated plasmid containing tandem lacO repeats by staining with an MPB-lacl fusion protein. HeLa cells were transfected with pSV2-dhfr-8.32 containing lacO tandem repeats and $24 \mathrm{~h}$ later were infected with 3 PFU per cell of vV5D4 expressing V5-tagged uracil DNA glycosylase. At $12 \mathrm{~h}$ after infection, cells were fixed, permeabilized, incubated with MBP-lacl and rabbit antibody to MBP (anti-MBP) and mouse monoclonal antibody to the V5 epitope (anti-V5) followed by Cy5conjugated donkey anti-mouse IgG and Texas red dye conjugated donkey anti-rabbit IgG. Cells were counterstained with Hoechst dye and analyzed by confocal microscopy. Colors: deep blue, Hoechst dye; red, Texas red; white, Cy5; light blue, overlap of Texas red and Cy5; yellow, overlap of Hoechst and Cy5.

minichromosome construct [32]. Moreover, our finding was similar to the 8-fold increase in net plasmid DNA reported by DeLange and McFadden [30]. There are several procedural differences that might account for the disparate results. One difference was the type of virus and cell used: Du and Traktman used mouse L cells infected with VAC, DeLange and McFadden principally used rabbit cells infected with myxoma virus or Shope fibroma virus and we used monkey or HeLa cells infected with VAC. A second difference was the method of DNA isolation. Whereas we and DeLange and McFadden proteinase digested whole cell lysates, Du and Traktman lysed cells with cold hypotonic buffer containing a non-ionic deter- gent and removed nuclei by sedimentation prior to DNA extraction. VAC DNA replication occurs in juxtanuclear factories and loss of high molecular weight protein-DNA complexes, especially those containing long head-to-tail plasmid DNA concatemers upon centrifugation is a concern. Indeed, Du and Traktman [32] reported that the presence of the telomere resolution sequence was required for high efficiency replication of linear minichromosomes and that only monomeric products were recovered. Further studies are needed to determine whether the cis-acting sequences in the linear minichromosomes are serving as origins of replication or as concatemer resolution sites or both. 


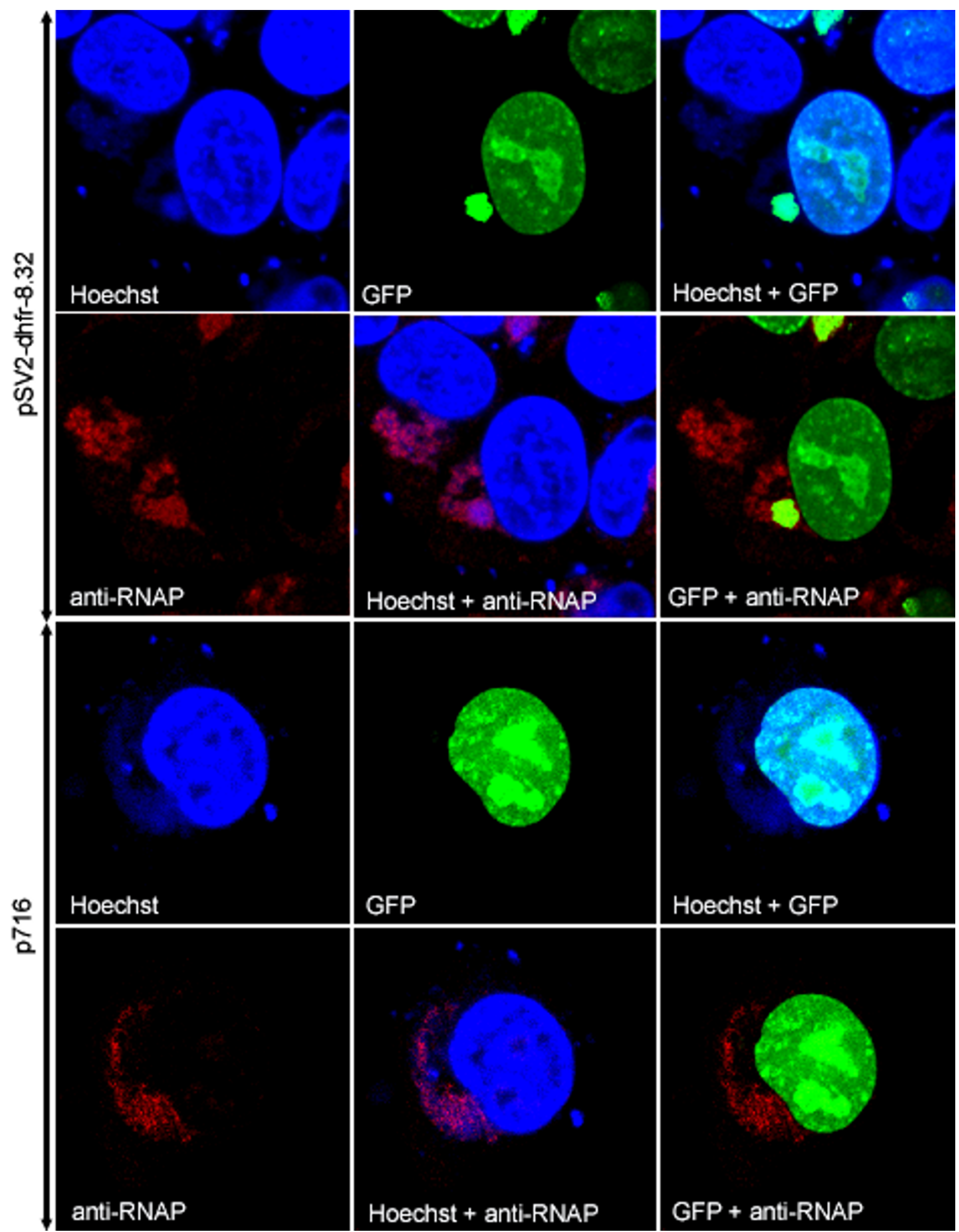

\section{Figure 4}

Intracellular localization of replicated plasmid containing tandem lacO repeats using a lacl-GFP fusion protein. HeLa cells were transfected with pSV2-dhfr-8.32 containing tandem lacO repeats (top 2 panels) or p7 6 control plasmid (bottom 2 panels) and infected with vGFP-lacl. At $12 \mathrm{~h}$ after infection, cells were fixed, permeabilized, and stained with antibody to VAC RNA polymerase (anti-RNAP), followed by Alexa 594-conjugated goat anti-rabbit lgG. Cells were then stained with Hoechst dye and analyzed by confocal microscopy. Blue, Hoechst; red, Alexa 594; and green, GFP fluorescence. 

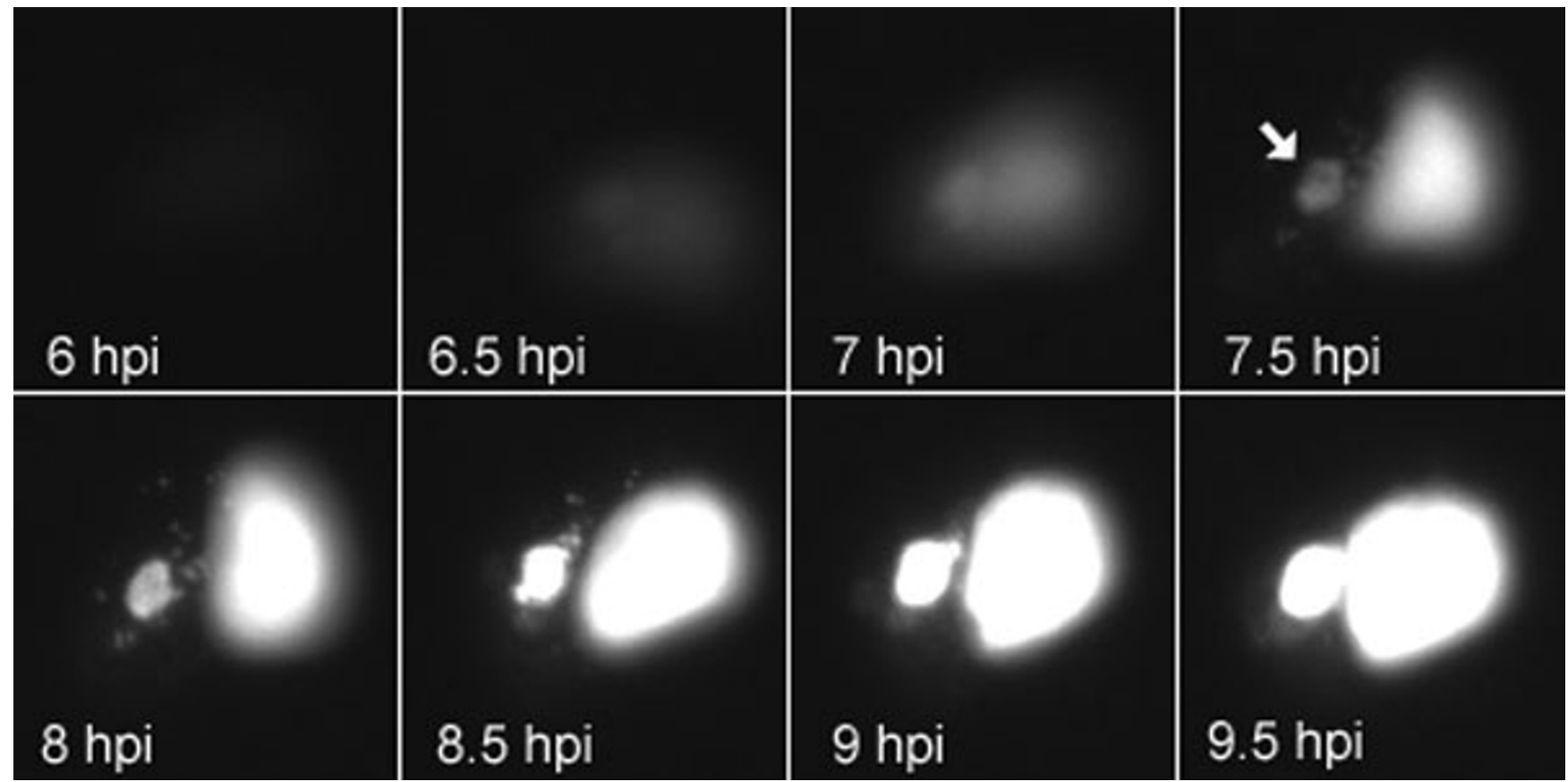

\section{Figure 5}

Visualization of plasmid replication in live HeLa cells. HeLa cells were transfected with pSV2-dhfr-8.32 containing tandem lacO repeats and infected with vGFP-lacl. Images were made at 5 min intervals starting at $5.5 \mathrm{~h}$ and ending at $10 \mathrm{~h}$ post infection.

Selected images at the indicated time points are shown starting at $6 \mathrm{~h}$ time point. Arrow at $7.5 \mathrm{~h}$ time point indicates cytoplasmic site of replicated plasmid.

The temporal coincidence of plasmid and viral DNA replication suggested that viral proteins were needed for each. Indeed, we found that each of the five trans-acting viral proteins known to be important for viral genome replication was similarly required for plasmid replication. Either none of these proteins have a sequence-specific role or some have dual roles and are also required for originindependent replication. The proteins also may have structural roles in assembling the replication complex, the existence of which is suggested by the interaction of A20 with the D4 and D5 proteins [45] and the co-purification of the A20, D4 and E9 proteins with a processive form of DNA polymerase $[46,47]$.

VAC cores containing genomic DNA and an early transcription system travel from the cell entry site along microtubules to the juxtanuclear area where synthesis of early viral proteins and DNA replication result in the formation of discrete factories [48]. It is believed that each factory arises from a single infectious particle [49]. It was interesting to determine whether plasmid replication occurred in factories or dispersed throughout the cell. To investigate this, we transfected cells with a plasmid containing multiple repeats of the E. coli lac $\mathrm{O}$, which tightly binds lacI. In one approach, the lacO DNA was located in discrete juxtanuclear regions by staining fixed and permeabilized cells with an MBP-lacI fusion protein followed by an antibody to MBP. The regions were identified as viral factories by Hoechst DNA staining and localization of the viral uracil DNA glycosylase, a protein required for replication of both plasmid and viral DNA. LacO DNA was not detected in the nucleus or in diffuse areas of the cytoplasm. A second approach involved the construction of a recombinant VAC that expresses a GFP-lacI fusion protein with a NLS to remove unbound protein from the cytoplasm. Again, the lacO DNA was found in viral factories identified with Hoechst staining and viral RNA polymerase antibody. The data suggest that for plasmid replication to occur, the DNA must be at the right place i.e. a site containing viral replication proteins. Presumably the plasmid diffuses into the factory region and is captured by DNA binding proteins. By taking time lapse images of live cells, plasmid DNA was detected in juxtanuclear sites at 6 to $7 \mathrm{~h}$ after infection and increased in intensity as the factories enlarged over the next several hours. Factory enlargement appeared to occur from within rather than by fusion of multiple small factories. We suspect that the latter might occur if higher multiplicities of virus were used. 
In contrast to the cytoplasmic replication of genome and plasmid DNA in VAC-infected cells, Sourvinos et al. [50] visualized nuclear replication of herpes simplex virus amplicons containing tetracycline operator sequence and Fraefel et al. [51] incorporated lac $\mathrm{O}$ sites into the genome of adenovirus associated virus and visualized discrete replication sites in the nucleus that fused to form larger structures. The latter study encouraged us to try to incorporate long tandem arrays of lacO repeats in the VAC genome, but they were unstable.

\section{Conclusion}

We described a sensitive and quantitative real-time PCR method of measuring plasmid replication in cells infected with VAC and demonstrated that origin-independent replication requires all known viral replication proteins. In addition, we visualized the plasmid in living and fixed cells by incorporating tandem lac $\mathrm{O}$ sequences and determined that replication occurred in cytoplasmic viral factories. This system should be useful for studying the mechanism and minimal requirements of poxvirus DNA replication.

\section{Methods \\ Cells, plasmids, and viruses}

RK-13, BS-C-1, BS-C-40, HuTK-143B, and HeLa cells were maintained in Eagle's minimal essential medium (EMEM; Quality Biologicals, Inc. Gaithersburg, MD) or Dulbecco's modified Eagle's medium (DMEM; Quality Biologicals, Inc.) containing $10 \%$ fetal bovine serum (FBS). A rabbit kidney cell line (RKD4R) stably expressing the VAC uracil DNA glycosylase and recombinant VAC vD4-ZG lacking a functional D4R gene [39] were gifts of F.G. Falkner. Plasmid pSV9 contains two copies of a $2.6 \mathrm{kbp}$ insert derived from the VAC concatemer junction and two copies of pUC13 DNA [33]. Linear minichromosomes containing $1.3 \mathrm{kbp}$ of VAC telomere sequences were prepared by ligation of snap cooled, EcoRI digested pSV9 essentially as described by Du and Traktman [32]. Ligation resulted in three products of $8 \mathrm{kbp}, 2.6 \mathrm{kbp}$ and $5.3 \mathrm{kbp}$. The $5.3 \mathrm{kbp}$ minichromosome fragment was isolated by gel electrophoresis and the Qiaex II gel extraction kit (Qiagen). Plasmid p716 [52] was kindly provided by A. McBride; plasmids pSV2-dhfr-8.32 and p3'SS dimer-Cl-EGFP [44] were gifts of A. Belmont. The temperature sensitive (ts) replication mutants Cts16, Cts24, Cts42, Cts25 with mutations in the I7, D5, E9 and B1 open reading frames, respectively were obtained from $\mathrm{R}$. Condit $[53,54]$; mut185 has a ts mutation in the A20 ORF [10].

\section{Antibodies}

Cy5-conjugated affinipure $\mathrm{F}(\mathrm{ab}) 2$ fragment of donkey anti-mouse IgG and Texas red dye conjugated affinipure $\mathrm{F}\left(\mathrm{ab}^{\prime}\right) 2$ of donkey anti-rabbit IgG were obtained from Jackson ImmunoResearch laboratories. Alexa Fluor 594 goat anti-rabbit IgG was from Molecular probes. New England Biolabs and Invitrogen supplied the rabbit antibody to MBP and mouse anti-V5 monoclonal antibody, respectively.

\section{Transfection, infection and isolation of DNA}

For experiments analyzed by real-time PCR, $0.1 \mu \mathrm{g}$ of p716 DNA and $3.9 \mu \mathrm{g}$ of salmon sperm carrier DNA were mixed with $10 \mu \mathrm{g}$ of lipofectamine 2000 (Invitrogen) and uninfected cells were transfected according to the manufacturer's instructions. After $24 \mathrm{~h}$, the cells were infected with VAC strain WR, vD4-ZG or a ts mutant at a multiplicity of 3 PFU per cell. Cells were then washed twice with Opti-MEM (Invitrogen) and overlaid with EMEM with $2.5 \%$ FBS. At various times, cells were harvested and the DNA isolated using the Qiamp DNA Blood Kit (Qiagen) according to the manufacturer's instructions. DNA was digested with restriction enzymes DpnI or Mbol (New England Biolabs).

\section{Southern blotting}

DNA $(2 \mu \mathrm{g})$ was digested with EcoRI and DpnI or MboI, resolved on a $0.8 \%$ agarose gel, and transferred to Immobilon-Ny+ (Millipore) transfer membrane. Southern blotting was carried out as described by Maniatis [55]. Plasmid DNA was detected with a DNA probe that was ${ }^{32}$ P-labeled using a random-priming kit (Invitrogen). Prehybridizations and hybridizations were carried out using Quik-Hyb (Stratagene) according to the manufacturer's recommendation. The blot was exposed to a Phosphor screen and data acquired on a Storm 860 PhosphoImager (Molecular Dynamics, Sunnyvale, CA) and quantified with ImageQuant software (Molecular Dynamics).

\section{Real-time PCR}

Oligonucleotides

P1

(5'CAACTAAATGTGCAAGCAATGTAATTC3') and P2 (5'CATCCTGCCCCTTGCTGT3') were designed with Primer Express software supplied by Applied Biosystems. Reactions were carried out using SYBR Green PCR master mix (Applied Biosystems), $10 \mu \mathrm{M}$ of each primer, and $1 \mathrm{ng}$ of DNA in a total volume of $50 \mu \mathrm{l}$ in an Applied Biosystems Prism 7900HT sequence detection system with v2.1.1 software. For amplification 40 cycles at $95^{\circ} \mathrm{C}$ for 15 $\mathrm{s}$ and $60^{\circ} \mathrm{C}$ for $60 \mathrm{~s}$ were used.

\section{Construction of recombinant viruses}

vGFP-lacI: the open reading frame that encodes GFP-lacI was cloned by PCR using primers 5'CAGGCTGCGCAACTGTTGGGAAGGGCGA3' and 5'AAAAGTACTAGCCTGGGGTGCCTAATGAGTGAGC3' with p3'SS dimer-Cl-EGFP [44] as a template. The PCR product was digested with $\mathrm{XhoI}$ and $\mathrm{ScaI}$ and then ligated to XhoI and StuI digested pSC59 [56] to form the plasmid pSC59gfplacI. BS-C-1 cells were infected with VAC strain 
WR at 0.05 PFU per cell for $1 \mathrm{~h}$ and then transfected with $2 \mu \mathrm{g}$ pSC59gfplacI using $10 \mu \mathrm{g}$ of Lipofectamine 2000. After $5 \mathrm{~h}$, the medium was replaced with EMEM plus $2.5 \%$ FBS and the incubation continued for 2 days. Cells were harvested and lysed, and the diluted lysates were used to infect HuTK- 143B cell monolayers. The cells were overlaid with medium containing low melting point agarose and $25 \mu \mathrm{g}$ of 5-bromodeoxyuridine per ml. After three rounds of plaque purification, the viral DNA was screened for the presence of the inserted DNA by PCR. The recombinant virus was propagated and titrated as described previously [57]. vV5D4: primers 5'ACTAGATACGTATAAAAAGGTATCTAATTTGATATAAT GGGTAAGCCTATCCCTAACCCTCTCCTCGGTCTCGATT CTACGAATTCAGTGACTGT3' and 5'CTCCTGGACGTAGCCTTCGGG3' and DNA from plasmid pER-GFP [21] were used to add a V5 tag to the VAC D4R gene. After double digestion of the PCR product and plasmid with SnaBI and SmaI, the products were ligated together to form the new plasmid pERV5-GFP. Approximately $10^{6}$ RKD4R cells were infected with vD4-ZG at a multiplicity of $0.05 \mathrm{PFU}$ per cell for $1 \mathrm{~h}$ at $37^{\circ} \mathrm{C}$. The infected cells were washed twice with Opti-MEM and transfected with $2 \mu \mathrm{g}$ of pERV5-GFP using $10 \mu \mathrm{g}$ of Lipofectamine 2000. After $5 \mathrm{~h}$, the transfection mixture was replaced with EMEM containing 2.5\% FBS, and the cells were harvested at $48 \mathrm{~h}$ in $0.5 \mathrm{ml}$ of EMEM-2.5\% FBS. Lysates were prepared by freezing and thawing the cells three times and sonicating them twice for $30 \mathrm{~s}$. Recombinant viruses that expressed GFP were plaque purified five times on RKD4R cells. The genetic purity of recombinant viruses was confirmed by PCR and sequencing. The recombinant virus was propagated and titrated as described previously [57].

\section{Construction and expression of MBP-lacl}

The lac repressor gene was PCR amplified using the following primers 5'CGGAATTCTCATCGGGAAACCTGTCGTGCCAGCTGC $3^{\prime}$ and 5'CGCGGATCCTAGTGAAACCAGTAACGTTATACG3' and template DNA from p3'SS dimer-Cl-EGFP. The amplified fragment was cloned into the BamHI and EcoRI sites of the expression vector pMal-c2x (New England Biolabs) resulting in the plasmid pMalc $2 x-l a c I$. Luria-Bertani medium (500 ml) supplemented with ampicillin (100 $\mu \mathrm{g} / \mathrm{ml})$ and glucose $(0.2 \% \mathrm{w} / \mathrm{v})$ was inoculated with $5 \mathrm{ml}$ of an overnight culture of the E. coli ER2507 (New England Biolabs) containing the recombinant pMalc2x-lacI plasmid. The culture was grown at $37^{\circ} \mathrm{C}$ to a cell density of 0.5 at $\mathrm{A} 600 \mathrm{~nm}$ and the expression of protein was induced for $2 \mathrm{~h}$ at $37^{\circ} \mathrm{C}$ by adding isopropyl- $\beta$-D-thiogalactopyranoside to a final concentration of $0.3 \mathrm{mM}$. The culture was then centrifuged at $4000 \times g$ for $20 \mathrm{~min}$ at $4{ }^{\circ} \mathrm{C}$. A cell extract was prepared using B-PER reagent
(Pierce) according to the manufacturer's recommendation and the protein purified using the pMAL protein fusion and purification kit (New England Biolabs).

\section{Confocal microscopy and live cell imaging}

Cells were plated on glass cover slips in 12 well plates and transfected with $1 \mu \mathrm{g}$ of pSV2-dhfr-8.32 using $5 \mu \mathrm{g}$ of Lipofectamine 2000. After $24 \mathrm{~h}$, cells were infected with recombinant VAC at 3 PFU per cell. At $12 \mathrm{~h}$ after infection, cells were fixed with cold 4\% paraformaldehyde in phosphate buffered saline (PBS) at room temperature for 20 min. Fixed cells were permeabilized for 5 min with PBS containing either $0.2 \%$ Triton X-100 at room temperature. Permeabilized cells were incubated with primary antibodies at a 1:100 dilution in 10\% FBS for $30 \mathrm{~min}$, washed with PBS three times, and then incubated with secondary antibody at a 1:100 dilution in 10\% FBS for $30 \mathrm{~min}$ at room temperature. After washing with PBS three times, cover slips were incubated with Hoechst dye for $10 \mathrm{~min}$ at room temperature to visualize DNA staining. Stained cells were washed extensively with PBS and cover slips mounted in $20 \%$ glycerol. Cellular fluorescence was examined under a Leica TCS NT inverted confocal microscope and images were overlaid using Adobe Photoshop version 5.0.2.

For live cell imaging, HeLa cells were plated at 80\% confluence onto TC3 dishes (Bioptechs, Inc.) and infected with 3 PFU of virus per cell on the next day. Cells were imaged by either confocal or video microscopy. For video microscopy, a Hammumatsu C5985 camera and controller were attached to a Leica DMIRBE inverted fluorescence microscope. Images were digitized using an IC-PCI video capture card (Coreco Imaging, Inc.) controlled by Image Pro Plus software. Cells were maintained on a heated TC3 stage (Bioptechs, Inc.) with the temperature set at $37^{\circ} \mathrm{C}$.

\section{Competing interests}

The author(s) declare they have no competing interests.

\section{Authors' contributions}

FDS participated in the design and coordination of the study, acquisition and analysis of data, and preparation of the manuscript. BM designed and coordinated the study, assisted in the data analyses and contributed to the preparation of the manuscript.

\section{Acknowledgements}

We thank Norman Cooper for invaluable assistance with cell culture, Owen Schwartz for helping in confocal microscopy and live cell imaging, and Mike Baxter for his assistance in real-time PCR. A. McBride and A. Belmont provided plasmids and $R$. Condit and F. Falkner donated mutant viruses and a cell line.

\section{References}

I. Moss B: Poxviridae: the viruses and their replication. In Fields Virology Volume 2. 4th edition. Edited by: Fields BN, Knipe DM and Howley PM. Philadelphia, Lippincott-Raven; 2001:2849-2883. 
2. Broyles SS: Vaccinia virus transcription. J Gen Virol 2003, 84:2293-2303.

3. Sridhar P, Condit RC: Selection for temperature-sensitive mutations in specific vaccinia virus genes: isolation and characterization of a virus mutant, which encodes a phosphonoacetic acid-resistant, temperature-sensitive DNA polymerase. Virology 1983, 1 28:444-457.

4. Traktman P, Sridhar P, Condit RC, Roberts BE: Transcriptional mapping of the DNA polymerase gene of vaccinia virus. J Virol | $984,49:|25-| 3 \mid$.

5. McDonald WF, Traktman P: Vaccinia virus DNA polymerase. In vitro analysis of parameters affecting processivity. J Biol Chem 1994, 269:31190-31197.

6. Jones EV, Moss B: Mapping of the vaccinia virus DNA polymerase gene by marker rescue and cell-free translation of selected RNA. J Virol 1984, 49:72-77.

7. Earl PL, Jones EV, Moss B: Homology between DNA polymerases of poxviruses, herpesviruses, and adenoviruses: nucleotide sequence of the vaccinia virus DNA polymerase gene. Proc Natl Acad Sci USA 1986, 83:3659-3663.

8. Challberg MD, Englund PT: Purification and properties of the deoxyribonucleic acid polymerase induced by vaccinia virus. J Biol Chem 1979, 254:78I2-78I9.

9. Punjabi A, Boyle K, DeMasi J, Grubisha O, Unger B, Khanna M, Traktman $P$ : Clustered charge-to-alanine mutagenesis of the vaccinia virus A20 gene: temperature-sensitive mutants have a DNA-minus phenotype and are defective in the production of processive DNA polymerase activity. I Virol 200I, 75: $12308-12318$

10. Ishii K, Moss B: Role of vaccinia virus A20R protein in DNA replication: construction and characterization of temperature-sensitive mutants. J Virol 200I, 75:1656-1663.

II. Evans E, Traktman P: Molecular genetic analysis of a vaccinia virus gene with an essential role in DNA replication. J Virol |987, 61:3|52-3162.

12. Evans $E$, Traktman P: Characterization of vaccinia virus DNA replication mutants with lesions in the D5 gene. Chromosome 1992, 102:S72-S82.

13. Evans E, Klemperer N, Ghosh R, Traktman P: The vaccinia virus D5 protein, which is required for DNA replication, is a nucleic acid-independent nucleoside triphosphatase. J Virol 1995, 69:5353-536|.

14. Traktman P, Anderson MK, Rempel RE: Vaccinia virus encodes an essential gene with strong homology to protein kinases. J Biol Chem 1989, 264:2|458-2|46I.

15. Rempel RE, Anderson MK, Evans E, Traktman P: Temperature-sensitive vaccinia virus mutants identify a gene with an essential role in viral replication. / Virol 1990, 64:574-583.

16. Rempel RE, Traktman P: Vaccinia virus BI kinase: phenotypic analysis of temperature-sensitive mutants and enzymatic characterization of recombinant proteins. I Virol 1992, 66:4413-4426

17. Banham $A H$, Smith $G L$ : Vaccinia virus gene $B I R$ encodes a $34-$ $\mathrm{kDa}$ serine/threonine protein kinase that localizes in cytoplasmic factories and is packaged into virions. Virology 1992 191:803-812.

18. Stuart DT, Upton C, Higman MA, Niles EG, McFadden G: A poxvirus-encoded uracil DNA glycosylase is essential for virus viability. J Virol 1993, 67:2503-25I2.

19. Upton C, Stuart DT, McFadden G: Identification of a poxvirus gene encoding a uracil DNA glycosylase. Proc Natl Acad Sci USA 1993, 90:4518-4522.

20. Millns AK, Carpenter MS, DeLange AM: The vaccinia virusencoded uracil DNA glycosylase has an essential role in viral DNA replication. Virology 1994, 198:504-5I3.

21. De Silva FS, Moss B: Vaccinia virus uracil DNA glycosylase has an essential role in DNA synthesis that is independent of its glycosylase activity: catalytic site mutations reduce virulence but not virus replication in cultured cells. J Virol 2003 77:159-166.

22. Garcia AD, Aravind L, Koonin EV, Moss B: Bacterial-type DNA holliday junction resolvases in eukaryotic viruses. Proc Natl Acad Sci USA 2000, 97:8926-8931.

23. Baroudy BM, Venkatesan S, Moss B: Incompletely base-paired flip-flop terminal loops link the two DNA strands of the vac- cinia virus genome into one uninterrupted polynucleotide chain. Cell 1982, 28:315-324.

24. Geshelin P, Berns KI: Characterization and localization of the naturally occurring cross-links in vaccinia virus DNA. J Mol Biol 1974, 88:785-796.

25. Baroudy BM, Moss B: Sequence homologies of diverse length tandem repetitions near ends of vaccinia virus genome suggest unequal crossing over. Nucleic Acids Res 1982, 10:5673-5679.

26. Moyer RW, Graves RL: The mechanism of cytoplasmic orthopoxvirus DNA replication. Cell I98I, 27:39I-40I.

27. Pogo BG, O'Shea M, Freimuth P: Initiation and termination of vaccinia virus DNA replication. Virology |98I, 108:24I-248.

28. Pogo BG: Changes in parental vaccinia virus DNA after viral penetration into cells. Virology 1980, 101:520-524.

29. Merchlinsky M, Moss B: Sequence-independent replication and sequence-specific resolution of plasmids containing the vaccinia virus concatemer junction: requirements for early and late trans-acting factors. In Cancer Cells 6: Eukaryotic DNA Replication Edited by: Kelly T and Stillman B. New York, Cold Spring Harbor Laboratory; 1 988:87-93.

30. DeLange AM, Reddy M, Scraba D, Upton C, McFadden G: Replication and resolution of cloned poxvirus telomeres in vivo generates linear minichromosomes with intact viral hairpin termini. J Virol 1986, 59:249-259.

31. Oliveira S, Costa JV: Replication of transfected plasmid DNA by cells infected with African swine fever virus. Virology 1995, 207:392-399.

32. Du S, Traktman P: Vaccinia virus DNA replication: two hundred base pairs of telomeric sequence confer optimal replication efficiency on minichromosome templates. Proc Nat Acad Sci USA 1996, 93:9693-9698.

33. Merchlinsky M, Moss B: Resolution of linear minichromosomes with hairpin ends from circular plasmids containing vaccinia virus concatemer junctions. Cell 1986, 45:879-884.

34. Merchlinsky M, Moss B: Nucleotide sequence required for resolution of the concatemer junction of vaccinia virus DNA. Virol 1989, 63:4354-4361.

35. Merchlinsky M: Resolution of poxvirus telomeres: processing of vaccinia virus concatemer junctions by conservative strand exchange. / Virol 1990, 64:3437-3446.

36. DeLange AM, McFadden G: Sequence-nonspecific replication of transfected plasmid DNA in poxvirus-infected cells. Proc Nat Acad Sci USA 1986, 83:614-618.

37. DeLange AM, McFadden G: Efficient resolution of replicated poxvirus telomeres to native hairpin structures requires two inverted symmetrical copies of a core target DNA sequence. J Virol 1987, 61:1957-1963.

38. Kane EM, Shuman S: Vaccinia virus morphogenesis is blocked by a temperature-sensitive mutation in the 17 gene that encodes a virion component. J Virol 1993, 67:2689-2698.

39. Holzer GW, Falkner FG: Construction of a vaccinia virus deficient in the essential DNA repair enzyme uracil DNA glycosylase by a complementing cell line. J Virol 1997, 71:4997-5002.

40. Tsukamoto T, Hashiguchi N, Janicki SM, Tumbar T, Belmont AS, Spector DL: Visualization of gene activity in living cells. Nat Cell Biol $2000,2: 87$ I-878

41. Tumbar T, Sudlow G, Belmont AS: Large-scale chromatin unfolding and remodeling induced by VPI6 acidic activation domain. I Cell Biol 1999, I 45: | 34I-I 354.

42. Straight AF, Belmont AS, Robinett CC, Murray AW: GFP tagging of budding yeast chromosomes reveals that protein-protein interactions can mediate sister chromatid cohesion. Curr Biol 1996, 6:1599-1608.

43. Belmont $A S$, Straight AF: In vivo visualization of chromosomes using lac operator-repressor binding. Trends Cell Biol 1998, 8: $121-124$.

44. Robinett CC, Straight A, Li G, Willhelm C, Sudlow G, Murray A, Belmont AS: In vivo localization of DNA sequences and visualization of large-scale chromatin organization using lac operator/repressor recognition. / Cell Biol 1996, I35:1685-1700.

45. Ishii K, Moss B: Mapping interaction sites of the A20R protein component of the vaccinia virus DNA replication complex. Virology 2002, 303:232-239.

46. Klemperer N, McDonald W, Boyle K, Unger B, Traktman P: The A20R protein is a stoichiometric component of the proces- 
sive form of vaccinia virus DNA polymerase. J Virol 200I, 75:12298- 12307.

47. Stanista $E$, Traktman $P$ : Insight into the role of UDG in vaccinia virus replication: characterization of two ts UDG mutants: ; McGill University, Montreal, Canada. ; 2004:7I.

48. Mallardo M, Leithe E, Schleich S, Roos N, Doglio L, Krijnse Locker J: Relationship between vaccinia virus intracellular cores, early mRNAs, and DNA replication sites. J Virol 2002, 76:5167-5।83.

49. Cairns HJF: The initiation of vaccinia infection. Virology 1960, I I:603-623.

50. Sourvinos G, Everett RD: Visualization of parental HSV-I genomes and replication compartments in association with ND 10 in live infected cells. EMBO / 2002, 21:4989-4997.

5I. Fraefel C, Bittermann AG, Bueler H, Heid I, Bachi T, Ackermann M: Spatial and temporal organization of adeno-associated virus DNA replication in live cells. J Virol 2004, 78:389-398.

52. Winokur PL, McBride AA: Separation of the transcriptional activation and replication functions of the bovine papillomavirus-I E2 protein. EMBO J 1992, I I:4IIII-4I I8.

53. Condit RC, Motyczka A: Isolation and preliminary characterization of temperature-sensitive mutants of vaccinia virus. Virology 198I, I I 3:224-24I.

54. Condit RC, Motyczka A, Spizz G: Isolation, characterization, and physical mapping of temperature-sensitive mutants of vaccinia virus. Virology 1983, I 28:429-443.

55. Maniatis T, Fritsch EF, Sambrook J: Molecular Cloning: A laboratory manual. New York, Cold Spring Harbor; 1982.

56. Chakrabarti S, Sisler JR, Moss B: Compact, synthetic, vaccinia virus early/late promoter for protein expression. Biotechniques 1997, 23:1094-1097.

57. Earl PL, Moss B, Wyatt LS, Caroll MW: Generation of recombinant vaccinia viruses. In Current protocols in molecular biology Volume 2. Edited by: Ausubel FM, Brent R, Kingston RE, Moore DD, Seidman JG, Smith JA and Struhl K. New York, Wiley Interscience; 1998:16.17.1-16.17.19.

Publish with Biomed Central and every scientist can read your work free of charge

"BioMed Central will be the most significant development for disseminating the results of biomedical research in our lifetime. "

Sir Paul Nurse, Cancer Research UK

Your research papers will be:

- available free of charge to the entire biomedical community

- peer reviewed and published immediately upon acceptance

- cited in PubMed and archived on PubMed Central

- yours - you keep the copyright
BioMedcentral 